\title{
On the difference in scattering behavior of cyclic and linear polymers in bulk
}

\author{
S. Gagliardi, V. Arrighi, ${ }^{a)}$ and R. Ferguson \\ Chemistry, School of Engineering and Physical Sciences, Heriot-Watt University, \\ Riccarton, Edinburgh EH14 4AS, United Kingdom
}

\author{
A. C. Dagger and J. A. Semlyen ${ }^{\text {b) }}$ \\ Department of Chemistry, University of York, Heslington, York Y010 5DD, United Kingdom \\ J. S. Higgins \\ Department of Chemical Engineering, Imperial College London, South Kensington Campus, \\ London SW7 2AZ, United Kingdom
}

(Received 22 April 2004; accepted 22 November 2004; published online 1 February 2005)

\begin{abstract}
It has been suggested that, due to topological constraints, rings in the melt may assume a more compact shape than Gaussian chains. In this paper, we exploit the availability of narrow fractions of perdeuterated linear and cyclic polydimethylsiloxane (PDMS) and, through the analysis of the small angle neutron scattering (SANS) profiles, demonstrate the difference in scattering properties of linear and cyclic PDMS molecules. As expected for Gaussian chains, for the H/D linear PDMS samples, log-log plots of the scattered intensity versus scattering vector $Q$ display a $Q^{(-2)}$ dependence. However, for H/D cyclic blends, the scaling exponent is higher than 2, as predicted by computer simulations reported in the literature. We show that cyclic molecules in bulk display the characteristic maximum in plots of scattered intensity versus $Q^{(-2)}$ that is expected on the basis of Monte Carlo calculations and from the Casassa equation [E. F. Casassa, J. Polym. Sci. A 3, 605 (1965)]. It is also shown that, for rings, the Debye equation [P. Debye, J. Appl. Phys. 15, 338 (1944)] is no longer appropriate to describe the SANS profiles of H/D cyclic blends, at least up to $M_{w} \approx 10000$. For these samples, the Casassa form factor gives a better representation of the SANS data and we show that this function which was developed for monodisperse cyclics is still adequate to describe our slightly polydisperse samples. Deviations from all above observations are noted for $M_{w}>11000$ and are attributed to partial contamination of cyclic samples with linear chains. The failure of both the Debye and the Casassa form factors could be due to contamination of the cyclic fractions by linear polymers or to a real conformational change. (C) 2005 American Institute of Physics. [DOI: 10.1063/1.1849162]
\end{abstract}

\section{INTRODUCTION}

The conformational and dynamic properties of cyclic polymers have generated considerable interest: the absence of chain ends leads to important changes on many physical properties, e.g., the molecular weight dependence of the glass transition, $T_{g}$, diffusion coefficients and radius of gyration, $R_{g}$, to name a few. While all experimental studies have dealt with the dilute solution behavior of cyclic molecules ${ }^{1-4}$ theoretical and computational work has been carried out on cyclics, in bulk. ${ }^{5-18}$ The general conclusion is that the conformation of rings in the melt differs significantly from that in solution, as a result of topological constraints active in concentrated systems but not in dilute solution.

The availability of narrow fractions of perdeuterated cyclic and linear polydimethylsiloxane (PDMS) (Refs. 19-21) has prompted us to perform a series of neutron scattering experiments in order to compare the conformational properties of cyclics and linear PDMS chains. A comparison be-

\footnotetext{
${ }^{a)}$ Electronic mail: V.Arrighi@hw.ac.uk

${ }^{b}$ Deceased.
}

tween the radii of gyration of cyclic and linear PDMS has been reported elsewhere ${ }^{22}$ while here we concentrate on the analysis of the scattering function and compare results with calculations and theoretical predictions.

It has been long established that in a dense system of linear chains such as in the melt, each chain is Gaussian and ideal. ${ }^{23}$ Thus the radius of gyration, $R_{g}$, of such chains is proportional to the square root of the number of segments $n$, i.e., to the square root of the polymerization index $N$ or, equivalently, the molecular weight $M$ :

$$
R_{g, \text { linear }} \propto n^{0.5} \propto N^{0.5} \propto M^{0.5} .
$$

Neutron scattering experiments on a few labeled (deuterated) chains in a melt of identical (hydrogenous) chains have convincingly shown that the chains are indeed ideal and Gaussian as expected by Flory. ${ }^{24,25}$

Although linking the two ends of a chain to form a ring may seem trivial, this constraint introduces an unexpected complexity into the theory of macromolecules, to date resolved only in the two limiting cases of rigid and very flexible rings. 
The radius of gyration for statistical molecules containing branches and rings has been calculated by Zimm and Stockmayer. ${ }^{26}$ Neglecting excluded volume effects and assuming the chain to be "reasonably" flexible, the result is given by

$$
R_{g, \text { cyclic }}^{2}=\frac{n b^{2}}{12},
$$

where $n$ is the number of segments in the molecule and $b$ is an effective bond length determined by the length and flexibility of the bonds. The same result had been previously obtained by Kramers. ${ }^{27}$

Using the same notation, for linear chains

$$
R_{g, \text { linear }}^{2}=\frac{n b^{2}}{6}
$$

leading to a striking difference between the mean square radius of gyration of rings and linear chains: $R_{g, \text { cyclic }}^{2}$ is half $R_{g, \text { linear. }}^{2}$

Analytical methods have been used by Casassa ${ }^{26,28}$ to calculate the form factor of cyclic polymers, $P(t)$, based on the assumption that the segmental pair distribution function differs from that of a Gaussian chain only in that the first and the last segments are joined together. This method leads to the following result for the form factor of a ring: ${ }^{28}$

$$
P(t)=\left(\frac{\sqrt{2}}{t}\right) D\left(\frac{t}{\sqrt{2}}\right)=\left(\frac{2}{t}\right) e^{-t^{2} / 4} \int_{0}^{t / 2} e^{x^{2}} d x,
$$

where $t=Q\left(R_{g, i}\right)\left(Q\right.$ being the scattering vector and $R_{g, i}$ the radius of gyration of component $i$ ) and $D(x)$ is the Dawson integral. This calculation and therefore Eq. (4) have been confirmed by other authors. ${ }^{9,29}$ Equation (4) predicts a maximum in a Krakty plot, $t^{2} P(t)$ versus $t$, at $t=2.1$ while for $t \Rightarrow \infty$, the normalized Kratky plots for cyclic and linear molecules should tend asymptotically to 1.0 and 2.0 , respectively, in a way that is consistent with the ratio between the corresponding $R_{g_{i}}$ values.

Monte Carlo (MC) calculations of the particle scattering functions $P(Q)$ for linear and cyclic PDMS containing up to 100 skeletal bonds ${ }^{9}$ show good agreement with the analytical calculations of Casassa and of Burchard and Schmidt, ${ }^{29}$ displaying a maximum in Kratky plots at around $t=2.0$. However, in the case of $\mathrm{MC}$, the maximum is less pronounced and for both cyclics and linear chains the limiting values for $t \Rightarrow \infty$ differ from the expected ones. This implies deviations from Gaussian statistics that are at the basis of the calculations in Refs. 26 and 28. Good agreement between MC calculations and experimental neutron scattering data on PDMS deuterated benzene solutions has been reported. ${ }^{9}$

There have been extensive computer simulations carried out on rings and these studies have lead to the conclusions that unconcatenated, unknotted cyclic molecules should adopt a more compact shape compared to linear Gaussian chains, due to topological constraints. ${ }^{11}$ For example, Pakula and Geyler ${ }^{12}$ reported a scaling exponent of 0.45 in the relationship between $R_{g}$ and the polymerization index, from computer simulations. Similar conclusions were drawn by Cates and co-workers first theoretically ${ }^{11}$ and then using computer simulations. ${ }^{5,17}$ The condition that rings remain neither knotted nor concatenated generates a topological constraint ${ }^{11}$ that is not completely screened out by the presence of a high density of other rings, as is the case for linear chains in bulk. The associated entropy loss is approximately equal to one degree of freedom per neighboring chain that the ring is prevented from threading. Additionally, there is an entropy penalty that prevents the ring from becoming too squashed, and the balance between these two opposite effects determines the cyclic conformation. The authors conclude that the Gaussian nature of the rings is not consistent with the topological constraints imposed by the requirement of unconcatenation; $R_{g}$ now varies with the polymerization in$\operatorname{dex} N$ as [see also Eq. (1)]

$$
R_{g, \text { cyclic }} \propto N^{v}
$$

with a scaling exponent $\nu=2 / 5=0.4$, intermediate between $\nu=1 / 2$ for Gaussian chains [Eq. (1)] and $\nu=1 / 3$ for segregated chains.

Computer simulations by Muller, Wittmer, and Cates ${ }^{5}$ on rings containing up to 512 monomers gave a scaling exponent $\nu=0.45$, in agreement with values reported by Pakula and Geyler ${ }^{12}$ and work of Brown and Szame ${ }^{15,16}$ and Brown, Lenczycki, and Szamel. ${ }^{18}$ In the limit of infinite molar mass $\nu=0.39 \pm 0.03$, in excellent agreement with theoretical predictions. ${ }^{11}$

Thus, small angle neutron scattering (SANS) experiments, in providing information on the radii of gyration of bulk polymers, should allow us to test whether, as expected from computer simulations, rings in the melt adopt more compact shapes than linear Gaussian chains. While we anticipate here that this is true, full analysis of the SANS determined molecular weight dependence of the radii of gyration is presented elsewhere. ${ }^{22}$ Here, through detailed analysis of the scattering profiles in different $Q$ ranges, we provide evidence for differences in the conformation of rings and linear chains.

\section{EXPERIMENT}

\section{A. Materials}

The hydrogenous cyclic poly(dimethyl siloxane)s were prepared using the ring/chain equilibration method of Chojnwski and Wilczek. ${ }^{30}$ This produced a polydisperse sample that was solution separated and then fractionated by preparative gel permeation chromatography (GPC) (see Refs. 31 and 32). The hydrogenous linear materials (which have trimethylsilyl end groups) were DC 200 series dimethylsiloxane polymers supplied by Dow Corning Ltd. Linear and cyclic deuterated polymers were synthesized using a procedure reported earlier. ${ }^{19-21}$ Similarly to the hydrogenous cyclic polymers, the deuterated materials were solution separated and, after fractionation using preparative GPC, a series of wellcharacterized narrow fractions of deuterated linear and cyclic polymers were obtained, with polydispersity in the range $1.03-1.25$. Due to the synthetic procedure adopted, the linear deuterated polymers present silanol end groups.

Molar mass values for the hydrogenous linear and cyclic materials used for the neutron scattering experiments were in 
TABLE I. Characteristics of cyclic PDMS samples used for neutron scattering experiments: $M_{w}$ is molar mass in $\mathrm{g} \mathrm{mol}^{-1}$ and PD stands for polydispersity $\left(M_{w} / M_{n}\right)$. Errors were calculated from the standard deviations on the GPC calibration curve.

\begin{tabular}{|c|c|c|c|c|c|}
\hline \multicolumn{3}{|c|}{ Hydrogenous cyclic PDMS } & \multicolumn{3}{|c|}{ Deuterated cyclic PDMS } \\
\hline LABEL & $M_{w}\left(\mathrm{~g} \mathrm{~mol}^{-1}\right)$ & PD & LABEL & $M_{w}\left(\mathrm{~g} \mathrm{~mol}^{-1}\right)$ & PD \\
\hline $20 \mathrm{HC}$ & $19800 \pm 2100$ & 1.04 & $15 \mathrm{DC}$ & $16300 \pm 1700$ & 1.08 \\
\hline $9 \mathrm{HC}$ & $8900 \pm 900$ & 1.12 & $11 \mathrm{DC}$ & $11100 \pm 1200$ & 1.11 \\
\hline $5 \mathrm{HC}$ & $4800 \pm 500$ & 1.06 & 9DC & $8600 \pm 900$ & 1.16 \\
\hline \multirow[t]{3}{*}{$3 \mathrm{HC}$} & $2800 \pm 300$ & 1.03 & $5 \mathrm{DC}$ & $4800 \pm 500$ & 1.13 \\
\hline & \pm & & $4 \mathrm{DC}$ & $3600 \pm 400$ & 1.14 \\
\hline & \pm & & $3 \mathrm{DC}$ & $2800 \pm 300$ & 1.18 \\
\hline
\end{tabular}

the range 2000-20 $000 \mathrm{~g} \mathrm{~mol}^{-1}$, as shown in Tables I and II. These were determined from GPC measurements, after calibration with linear or cyclic PDMS narrow standards. For the deuterated polymers the same calibration curves were used but $M_{w}$ values were corrected to account for the difference between the molecular weights of the hydrogenous and deuterated monomers.

\section{B. SANS measurements}

The conformational and thermodynamic properties of linear and cyclic PDMS in bulk were investigated in a series of experiments carried out on the small angle neutron scattering diffractometers LOQ (ISIS, Rutherford Appleton Laboratory, UK) and D22 (Institut Laue Langevin, France).

On LOQ, incident neutrons with wavelengths in the range $2.2<\lambda<10 \AA$ were used, covering a $Q$ range $0.008-0.25 \AA^{-1}$. The raw data were radially averaged about the incident beam direction, the resulting scattered intensities were then corrected for sample transmission and thickness, and the scattering from the empty cell was subtracted using standard procedures. ${ }^{33}$ The differential scattering cross section per sample unit volume, $\partial \Sigma(Q) / \partial \Omega$, in units of $\mathrm{cm}^{-1}$, was obtained after normalization with a polystyrene standard [here $Q=4 \pi / \lambda(\sin \theta / 2)$ where $\lambda$ is the neutron wavelength and $\theta$ is the scattering angle].

On D22, two different configurations were used with a wavelength of $6 \AA$ and sample-detector distances of 1.5 and $8 \mathrm{~m}$, in order to cover the $Q$ range $0.0054-0.60 \AA^{-1}$. Standard ILL procedures were employed, using water as a secondary standard in order to normalize the radially averaged data, after subtracting the scattering from the empty cell. In both cases SANS measurements were carried out at $298 \mathrm{~K}$ using a temperature controlled sample holder.
For the purpose of evaluating the level of incoherent scattering in H/D PDMS mixtures, three randomly labeled H/D PDMS copolymers of different composition were prepared. The volume fraction of the hydrogenated component, determined by NMR measurements, was $0.44,0.72$, and 0.91 . The scattering patterns from these random H/D copolymers no longer contain information on the chain conformation and they allow estimates of the level of incoherent background. For LOQ, the SANS data of the random H/D copolymers were compared to the expected curves calculated from the volume fraction weighted sum of the scattered intensities of pure H-PDMS and D-PDMS samples. In this case the agreement between calculated curves and experimental data was found to be excellent (see Fig. 1 in Ref. 22). The random copolymers were not available at the time of the D22 experiment. In these measurements, the amount of incoherent scattering to be subtracted need to be accurately determined: the scattered intensity is very low in the high $Q$ region. Determination of the incoherent background from the volume fraction-weighted sum of the scattered intensities of pure H-PDMS and D-PDMS samples leads to slight overestimation and so no attempts were made to subtract calculated values prior to fitting: the incoherent background was evaluated during data fitting by multiplying the H-PDMS SANS curve using a concentration dependent fitting parameter.

\section{RESULTS AND DISCUSSION}

In the framework of the mean-field random phase approximation (RPA) derived by deGennes ${ }^{34}$ and Binder, ${ }^{35}$ the coherent differential scattering cross section, expressed in units of $\mathrm{cm}^{-1}$, for an H/D mixture of two identical polymers, differing only in the level of isotopic substitution, is given by

TABLE II. Characteristics of linear PDMS samples used for neutron scattering experiments: $M_{w}$ is molar mass in $\mathrm{g} \mathrm{mol}^{-1}$ and PD stands for polydispersity $\left(M_{w} / M_{n}\right)$. Errors were calculated from the standard deviations on the GPC calibration curve.

\begin{tabular}{|c|c|c|c|c|c|}
\hline \multicolumn{3}{|c|}{ Hydrogenous linear PDMS } & \multicolumn{3}{|c|}{ Deuterated linear PDMS } \\
\hline LABEL & $M_{w}\left(\mathrm{~g} \mathrm{~mol}^{-1}\right)$ & $\mathrm{PD}$ & LABEL & $M_{w}\left(\mathrm{~g} \mathrm{~mol}^{-1}\right)$ & PD \\
\hline $10 \mathrm{HL}$ & $9700 \pm 1300$ & 1.03 & $12 \mathrm{DL}$ & $11800 \pm 1600$ & 1.11 \\
\hline \multirow[t]{2}{*}{$2 \mathrm{HL}$} & $2000 \pm 300$ & 1.07 & $2.5 \mathrm{DL}$ & $2500 \pm 300$ & 1.20 \\
\hline & \pm & & $2 \mathrm{DL}$ & $2000 \pm 300$ & 1.18 \\
\hline
\end{tabular}




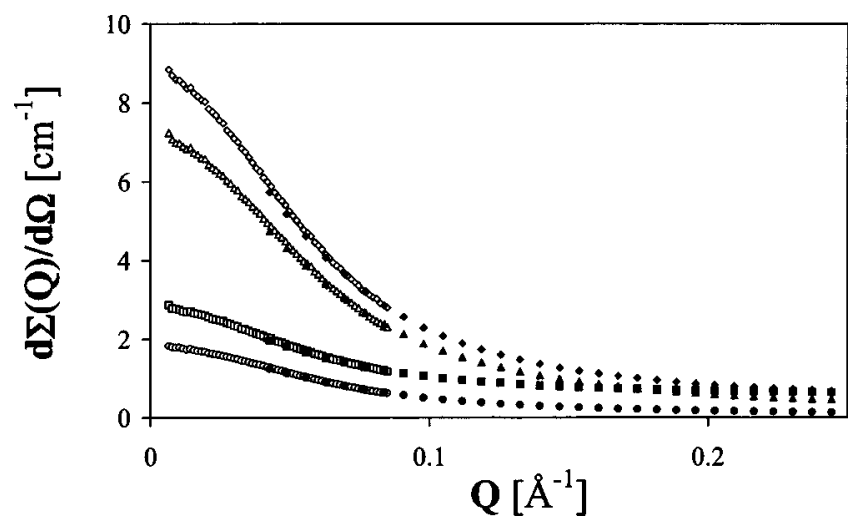

FIG. 1. SANS data of sample $10 \mathrm{HL}-12 \mathrm{DL}$ at volume fraction $\phi_{\mathrm{H}}=0.05$ (circles), 0.27 (triangles), 0.65 (diamonds), and 0.94 (squares) collected at D22 using both the small angle (symbols) and the large angle detectors (filled symbols).

$$
\frac{\partial \Sigma(Q)}{\partial \Omega}=\frac{(\Delta b)^{2}}{v_{0}}\left[\frac{1}{\phi_{\mathrm{H}} z_{\mathrm{H}} P_{\mathrm{H}}(Q)}+\frac{1}{\phi_{\mathrm{D}} z_{\mathrm{D}} P_{\mathrm{D}}(Q)}-2 \chi\right]^{-1},
$$

where $\Delta b$ gives the difference in scattering lengths of the hydrogeneous and deuterated monomers, $z_{i}$ and $\phi_{i}$ define the degree of polymerization and volume fraction of polymer $i$, and the subscripts $\mathrm{H}$ and $\mathrm{D}$ indicate the hydrogenous and deuterated components, respectively. Accounting for the slightly different volumes of the $\mathrm{H}$ and $\mathrm{D}$ monomers, $v_{\mathrm{H}}$ and $v_{\mathrm{D}}, \Delta b$ is

$$
\Delta b=v_{0}\left(\frac{b_{\mathrm{H}}}{v_{\mathrm{H}}}-\frac{b_{\mathrm{D}}}{v_{\mathrm{D}}}\right),
$$

where $v_{0}$ is the reference volume, $v_{0}=\sqrt{v_{\mathrm{H}} v_{\mathrm{D}}}$, and $b_{i}$ the scattering length.

The form factors, $P_{\mathrm{H}}(Q)$ and $P_{\mathrm{D}}(Q)$ in Eq. (6) model the change of the scattered intensity with scattering vector $Q$; appropriate analytical forms are available, depending on the system under study. For monodisperse Gaussian chains, the form factors $P_{i}(Q)$ in Eq. (6) are described by the Debye equation $^{34}$

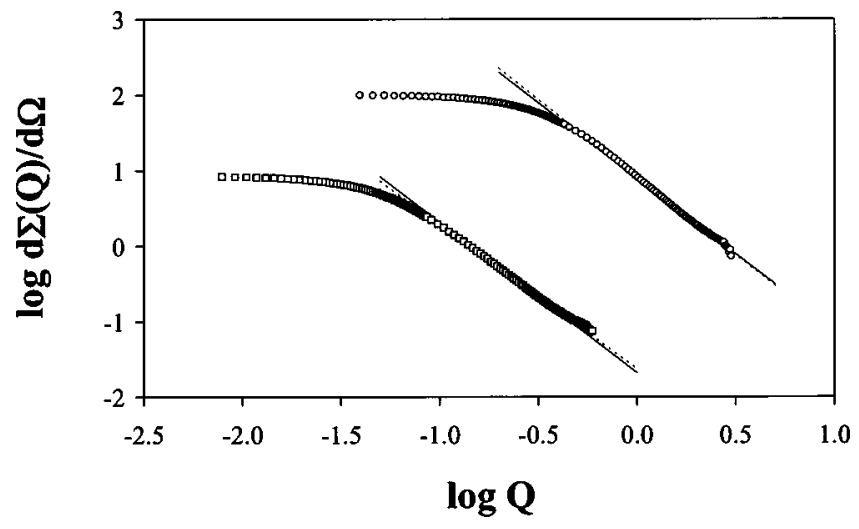

FIG. 2. Scattered intensity on a log-log scale for samples 9HC-11DC (circles) and 10HL-12DL (squares) at volume fraction $\phi_{\mathrm{H}}=0.66$ and 0.65 , respectively. The dashed lines indicate the experimental slopes at high $Q$ ( -2.07 and -1.92 , respectively). A $Q^{-2}$ dependence is shown for comparison (continuous lines). Data corresponding to 9HC-11DC were shifted along the axes for clarity.

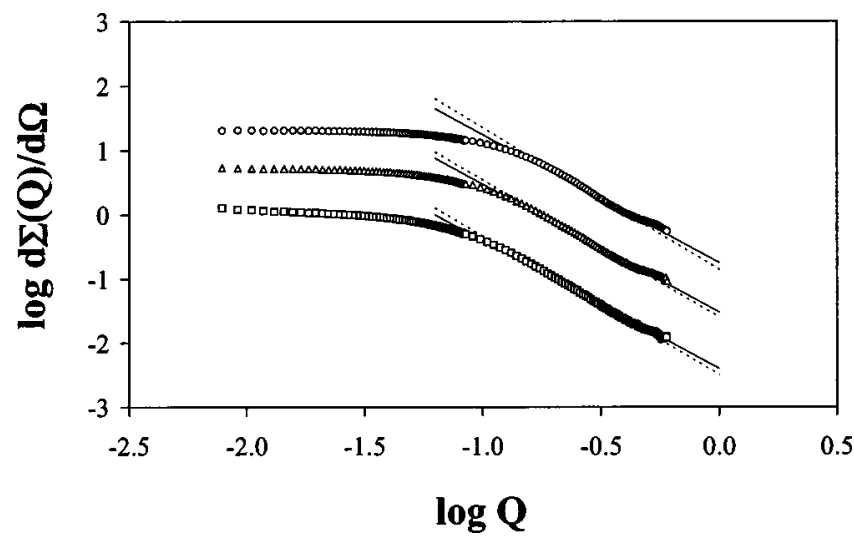

FIG. 3. Scattered intensity on a log-log scale for samples 9HC-3DC at volume fraction $\phi_{\mathrm{H}}=0.04$ (squares), 0.28 (triangles), and 0.66 (circles). The dashed lines indicate the experimental slopes at high $Q(-2.19)$. A $Q^{-2}$ dependence is shown for comparison (continuous lines). Data corresponding to $\phi_{\mathrm{H}}=0.28$ and 0.66 were shifted along the axes for clarity.

$$
P_{i}(Q)=\left(\frac{2}{Q^{4} R_{g, i}^{4}}\right)\left(Q^{2} R_{g, i}^{2}-1+e^{\left(-Q^{2} R_{g, i}^{2}\right)}\right)
$$

which applies to a monodisperse system. For a sample with polydispersity index $u=\left(M_{w} / M_{n}\right)-1$, a modified Debye function is often used to fit the experimental SANS data: ${ }^{36}$

$$
P(Q)=\frac{2}{(u+1) \gamma^{2}}\left[(1+u \gamma)^{-1 / u}-1+\gamma\right],
$$

where $\gamma=R_{z}^{2} Q^{2} /(1+2 u)$, with $R_{z}$ being the $z$-average radius of gyration.

Double logarithmic plots, i.e., $\ln [\partial \Sigma(Q) / \partial \Omega]$ versus $\ln (Q)$, provide useful information on the $Q$ dependence of the scattered intensity and aid selection of model functions. On the basis of Eqs. (8) and (9), it should be possible to distinguish three regions in the SANS curves: (a) at low $Q$, the scattered intensity is expected to reach a constant value that depends on blend composition, $M_{w}$ and the interaction parameter $\chi$, (b) in the intermediate $Q$ range, the SANS profiles decay exponentially while (c) at high $Q$, the curve follows a $Q^{-2}$ dependence. Thus, while Gaussian coils display a

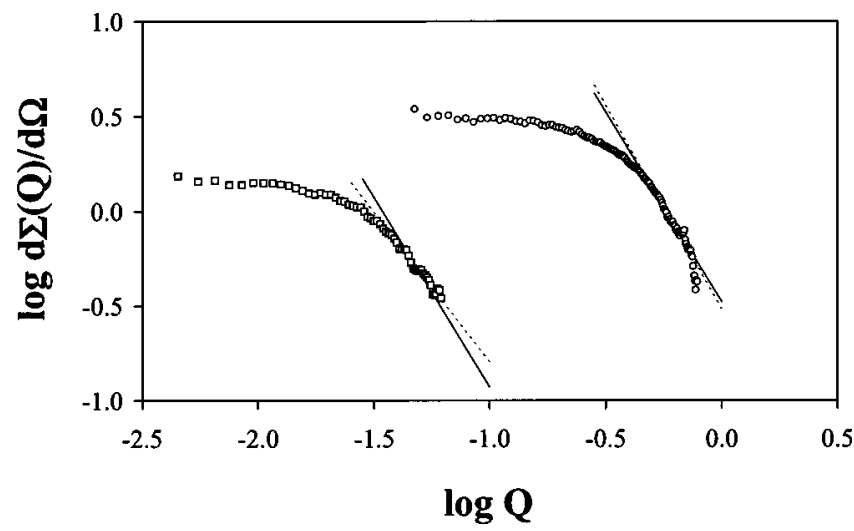

FIG. 4. Scattered intensity on a log-log scale for samples 3HC-4DC (circles) and 2HL-2DL (squares) at volume fraction $\phi_{\mathrm{H}}=0.51$ and 0.52 , respectively. The dashed lines indicate the experimental slopes at high $Q$. A $Q^{-2}$ dependence is shown for comparison (continuous lines). Data were shifted along the axes for clarity. 


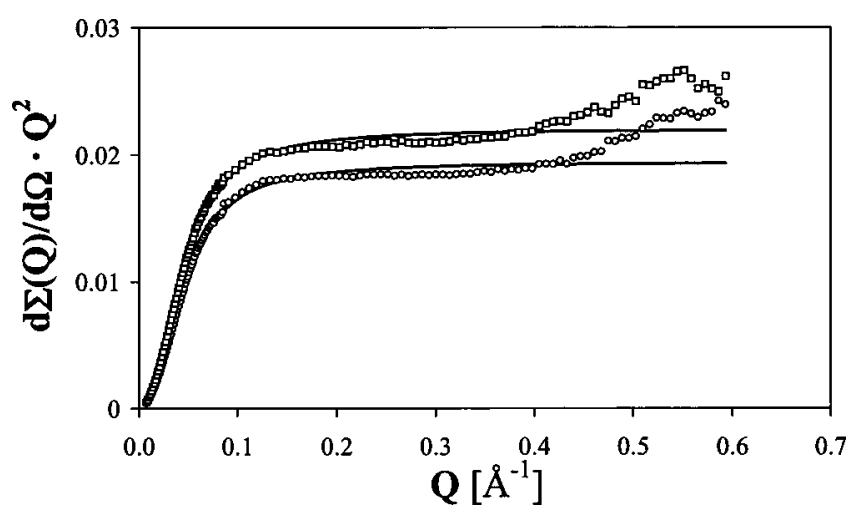

FIG. 5. Kratky plot for the 10HL-12DL blend at volume fraction $\phi_{\mathrm{H}}$ $=0.27$ (circles) and 0.65 (squares) along with calculated lines (see text for details).

$Q^{-2}$ dependence, at high $Q$, any deviation from this behavior should be indicative of non-Gaussian statistics.

In the case of the D22 data, overlap between the scattered intensity of H/D linear PDMS samples from the two detectors was excellent and could be achieved without any data manipulation as shown in Fig. 1. This procedure widened considerably the experimental $Q$ range, making it possible to explore the range of validity of the Debye equation. As shown in Fig. 2, the linear PDMS blend (10HL-12DL, where HL is hydrogenous linear and DL is deuterated linear) data follow the expected $Q^{-2}$ dependence at high $Q$ (for $\phi_{\mathrm{H}}=0.65$ in Fig. 2). The two cyclic blends studied on D22 behave differently: the high $Q$ slope for the 9HC-3DC (9 hydrogenous cyclic-3 deuterated cyclic) (Fig. 3) blend is lower than 2 (slope $=-2.19 \pm 0.01)$ at all concentrations, suggesting that cyclics are more compact than linear chains. This behavior is dependent upon the sample molecular weight since, for 9HC-11DC blends, the slope changes from $(-2.61 \pm 0.01)$ to $(-2.07 \pm 0.01)$ with increasing content of the hydrogenous component from $\phi_{\mathrm{H}}=0.05$ to $\phi_{\mathrm{H}}=0.66$ (data for $\phi_{\mathrm{H}}=0.66$ shown in Fig. 2). This implies swelling of the hydrogenous cyclic PDMS with increasing concentration and may be the result of contamination of the high $M_{w}$ cyclic fractions with linear chains. This result cannot be confirmed using data for the high molecular weight blends from LOQ, e.g., $20 \mathrm{HC}-15 \mathrm{DC}$, as the $Q$ range is too narrow to draw

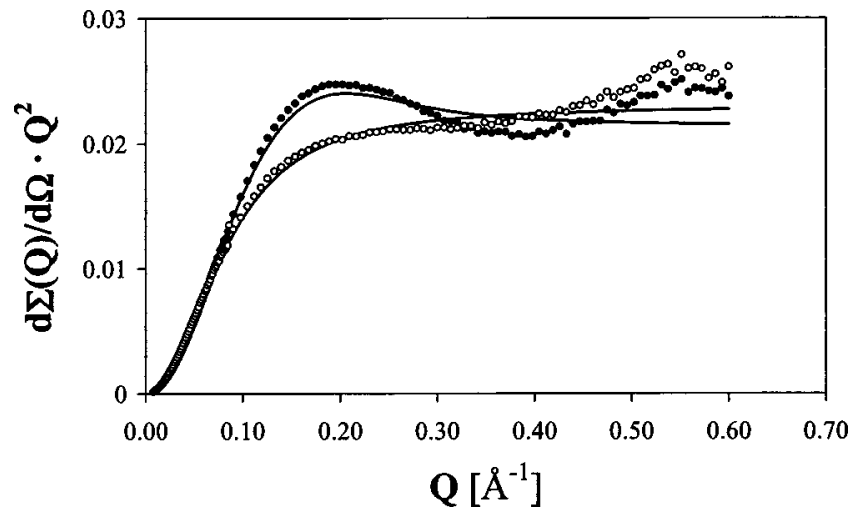

FIG. 6. Kratky plot for the blends 10HL-2.5DL (circles) and 9HC-3DC (filled circles) at volume fraction $\phi_{\mathrm{H}}=0.66$ and 0.68 , respectively, along with calculated lines (see text for details).

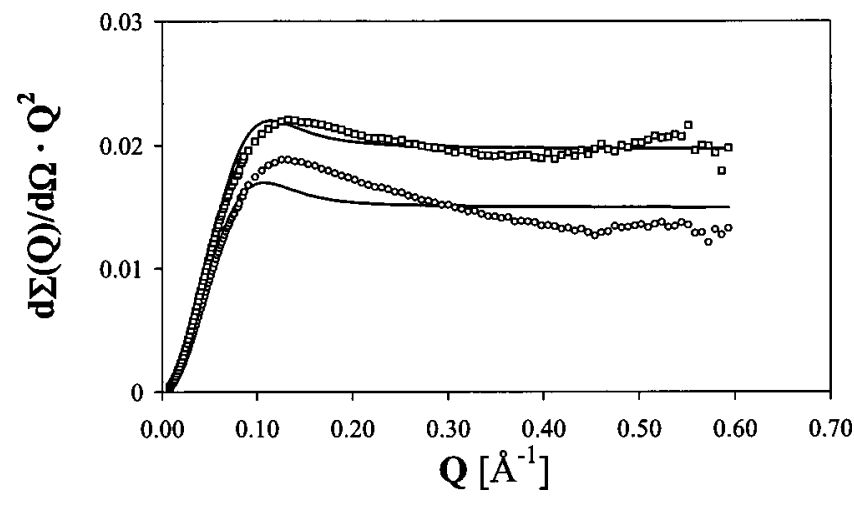

FIG. 7. Kratky plot for the $9 \mathrm{HC}-11 \mathrm{DC}$ blend at volume fraction $\phi_{\mathrm{H}}=0.26$ (circles) and 0.66 (squares) along with calculated lines (see text for details).

definite conclusions on the $Q$ dependence in the high $Q$ region. The LOQ data from the main detector extend up to $0.25 \AA^{-1}$, and in this region, the determination of the slope is very sensitive to the $Q$ range chosen to fit the data. Only indicative values can be obtained: in the melt, cyclics seem to be more compact than linear chains, as shown, for example, by comparing 3HC-4DC and 2HL-2DL in Fig. 4.

In the high $Q$ limit, $Q \geqslant\left(5 / R_{g}\right)$, Eq. (8) [once combined with Eq. (6) taking $\chi=0$ ] can be approximated to

$$
\frac{\partial \Sigma(Q)}{\partial \Omega}=\frac{2(\Delta b)^{2} \phi_{\mathrm{H}} \phi_{\mathrm{D}} N_{A} \rho M}{m^{2} Q^{2} R_{g}^{2}},
$$

where $\rho$ is the density, $N_{A}$ is Avogadro's number, and $m$ is the monomer molecular weight. Thus when the scattered intensity is plotted in the Kratky form, i.e., $\partial \Sigma(Q) / \partial \Omega \cdot Q^{2}$ versus $Q$, it should display a plateau. ${ }^{37}$ Deviations from this behavior have been observed for branched, star, and ring polymers: these structures should produce a peak in $\partial \Sigma(Q) / \partial \Omega \cdot Q^{2}$ before reaching an asymptotic value. Often the signal at high $Q$ is weak, i.e., the scattering intensity is low: good statistics and careful background measurements are essential for analysis of the high $Q$ region.

Once again the D22 data obtained by overlapping SANS data from the two detectors, offer an appropriately wide $Q$ range to allow comparison of the experimental and calculated scattered intensities, in a Kratky representation. In Figs. 5-8 the experimental scattered intensity (symbols) is plotted

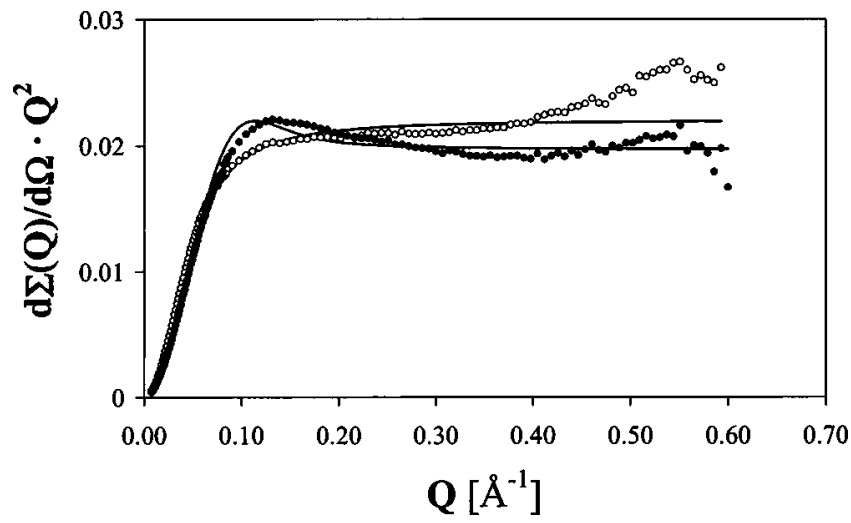

FIG. 8. Kratky plot for the blend 10HL-12DL (circles) and 9HC-11DC (filled circles) at volume fraction $\phi_{\mathrm{H}}=0.65$ and 0.66 , respectively, along with calculated lines (see text for details). 


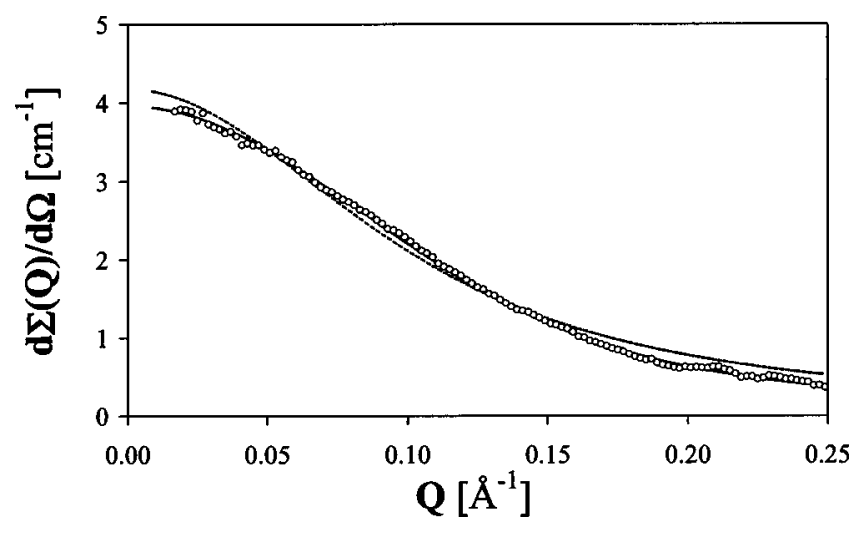

FIG. 9. Experimental data and best fitting obtained for the blend 5HC-5DC at $\phi_{\mathrm{H}}=0.52$ using either the Debye (dashed line) or the Casassa (continuous line) form factor. All fits were performed by letting both interaction parameter and radius of gyration vary.

and compared to the calculated $\partial \Sigma(Q) / \partial \Omega \cdot Q^{2}$ (lines) using form factors consistent with the topology of the blend components. As shown in Fig. 5 for 10HL-12DL, the agreement between calculated and experimental curves is very good for linear blends. The 9HC-3DC blend at $\phi_{\mathrm{H}}=0.66$ reported in Fig. 6 displays the expected peak whose maximum falls at a $Q$ value close to that calculated by the Casassa form factor.

However, pronounced deviations are observed for larger cyclics, as shown in Fig. 7 for 9HC-11DC. We note that when both blend components are considered as rings, i.e., the Casassa form factor is used in the calculations of $\partial \Sigma(Q) / \partial \Omega$ for both $\mathrm{H}$ and $\mathrm{D}$ components, there are strong deviations between experimental data and calculated curves (Fig. 7). Nevertheless, the presence of cyclics in 9HC-11DC is evidenced by the weak maximum in the Kratky plots, which is clearly noticeable when linear and cyclic blends of similar molecular weight are compared (Figs. 6 and 8). The results in Fig. 7 are then consistent with observations made earlier from the log-log plots, namely, that slope values are composition dependent.

Kratky plots are extensively used to investigate the effect of local chain structure and, as shown here, chain architecture. Effects of microstructure and chain stiffness are usually evident as deviations from the Debye equation and therefore manifest themselves in the Kratky plots. This is the

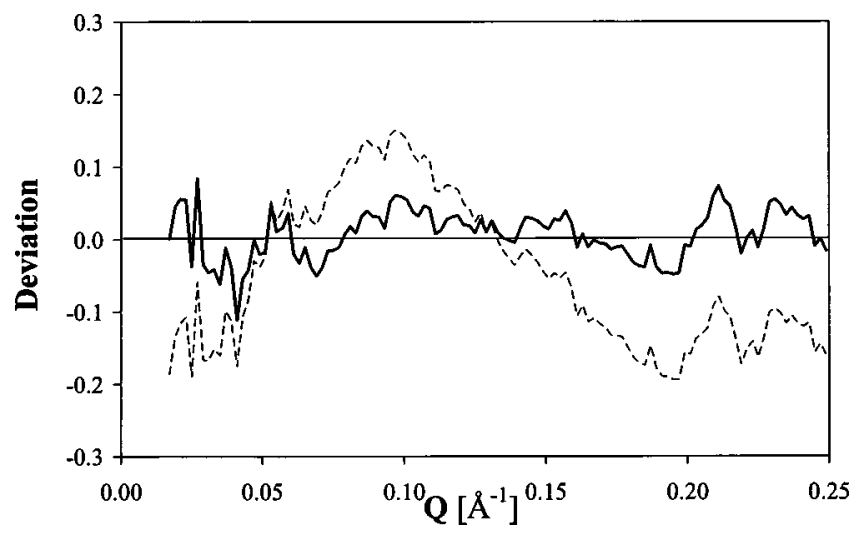

FIG. 10. Deviations between experimental data and best fit obtained for the 5HC-5DC sample at $\phi_{\mathrm{H}}=0.52$ using either the Debye (dashed line) or the Casassa (continuous line) form factor.

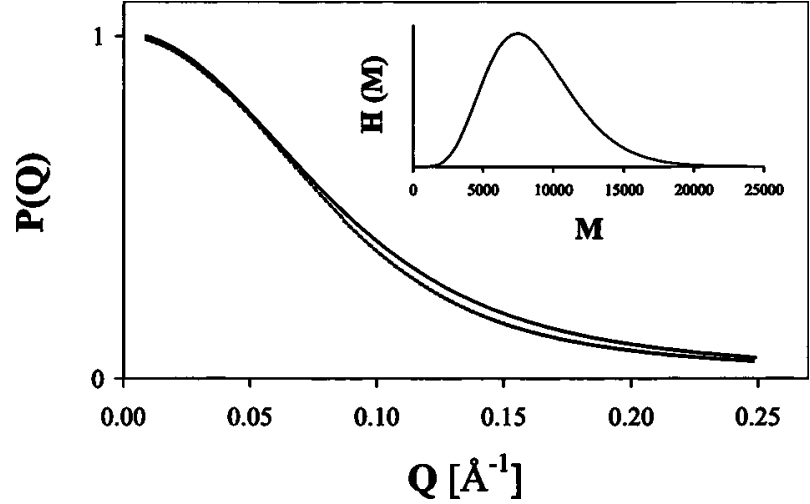

FIG. 11. Calculated $P(Q)$ obtained using the Casassa form factor (dashed line) and convoluting it with the Zimm-Schulz distribution (continuous line) for sample 9DC, characterized by a polydispersity 1.16. The Zimm-Schulz distribution is reported in the inset.

case for many polymeric systems including polycarbonate ${ }^{38}$ and poly(methyl methacrylate). ${ }^{39}$ It should however be noted that, in special circumstances, compensation effects may lead to appearance of a Kratky plateau. This is the case for polystyrene which shows distinct scattering behavior, depending on deuteration. ${ }^{38,40}$ In this case the extended Kratky plateau observed when both backbone and side groups contribute to the scattering is due to compensation between the rodlike signal (observed when using polystyrene samples, deuterated in the backbone) and the side groups. Unfortunately, measurements similar to those carried out on polystyrene are not possible for PDMS due to the inability to alter the contrast through selective deuteration. However, while the example of polystyrene clearly illustrates that both cross section and chain rigidity are important in determining the scattering at short distances, it is unlikely to be of relevance here. This argument rests on the belief that, compared to the poly(dimethyl siloxane) samples investigated here, polystyrene is a relatively rigid polymer with a large side group. Further analysis and/or calculations are required to prove whether the difference in chain stiffness (as indicated by the characteristic ratio) is sufficient to support the argument made here.

All data presented in Figs. 5-8 after reaching an asymptotic value display departure at higher $Q$. This is be-

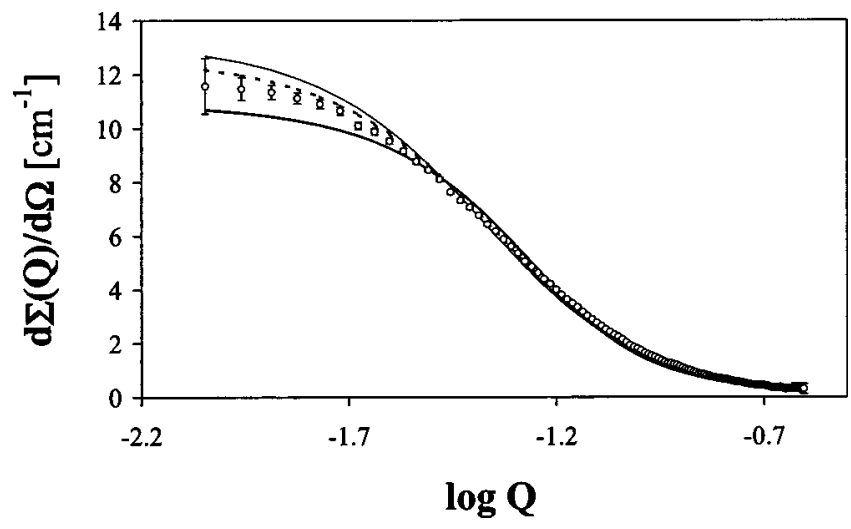

FIG. 12. Experimental data and best fitting obtained for the blend 20HC$15 \mathrm{DC}$ at $\phi_{\mathrm{H}}=0.26$ using the Debye (thin black line) or the Casassa (thick black line) form factor for both components, and using the Casassa for $15 \mathrm{DC}$ and the Debye equation for $20 \mathrm{HC}$ (dashed line). 
cause at shorter distances the SANS data probe the molecular structure of the polymer chains: $:^{37}$ a transition from a $1 / Q^{-2}$ (asymptote) to $1 / Q$ (linear) dependence of $\partial \Sigma(Q) / \partial \Omega$ is expected and it is qualitatively reproduced by our data. Although the persistence length of the chain could be evaluated from the $Q$ value corresponding to the crossover, ${ }^{37}$ for the data shown here the $Q$ range does not extend to sufficiently high $Q$ values to warrant this procedure.

It is perhaps remarkable that an extended plateau is observed for linear PDMS samples up to very high $Q$ values. This feature of the PDMS chains has already been noted by Beaucage et $a .^{41}$ who reported that the scattering curves of linear PDMS samples retain Gaussian scaling up to high $Q$ values. Good agreement was observed with the RPA function and the Debye equation (Fig. 1 in Ref. 41) over a wide $Q$ range, comparable to that reported here. This behavior is likely to be a result of the high flexibility of the PDMS chains and other flexible polymers such as poly(ethylene oxide) display similar properties. ${ }^{42}$

The Casassa equation provides an analytical function that can be used to fit the experimental SANS data. In this paper this function has been applied to model the scattered intensities measured for isotopic blends of cyclic molecules. The experimental SANS data were analyzed using Eq. (6) and the appropriate form factor, according to the blend topology. Since $M_{w}$ and $M_{w} / M_{n}$ are known from GPC measurements, there are only three unknown parameters to be determined: the radii of gyration of the two components and the interaction parameter.

In Fig. 9 an example of data fitting for a cyclic blend containing low molecular weight samples is reported: it is evident that the two form factors, i.e., Debye [Eq. (9)] and Casassa [Eq. (4)], are not interchangeable and it is not possible to fit the data of the cyclic blends using the Debye equation. This is further illustrated in Fig. 10 were we report the deviation between experimental data and fits: while in the case of fits performed using the Casassa equation, deviations are random throughout the $Q$ range, systematic changes are observed when using the Debye equation.

As discussed earlier, the SANS profiles are affected by the sample polydispersity and functions that account for this effect have been developed for various polymer structures. Unfortunately, an analytical function similar to Eq. (9) is not available for rings, and the Casassa form factor [Eq. (4)] is rigorously applicable only to monodisperse samples. However, it is possible to estimate the effect of polydispersity on the SANS profiles by considering that Eq. (9) is the result of convoluting Eq. (8) and a particle size distribution function, such as the Zimm-Schultz distribution: ${ }^{36,43,44}$

$$
H(M)=\frac{1}{\Gamma(s+1)}\left(\frac{s+1}{M_{w}}\right)^{s+1} M^{s} e^{\left[-(s+1) M / M_{w}\right]},
$$

where $s$ is the reciprocal $\left(u^{-1}\right)$ of the polydispersity index $u=\left(M_{w} / M_{n}-1\right)$, and $\Gamma$ is the so-called Gamma function:

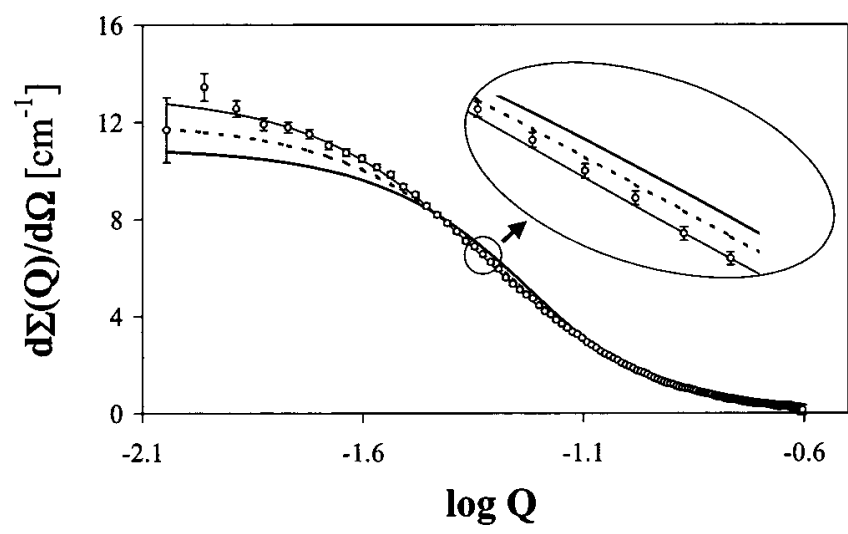

FIG. 13. Experimental data and best fitting obtained for the blend 20HC$15 \mathrm{DC}$ at $\phi_{\mathrm{H}}=0.52$ using either the Debye (thin line) or the Casassa (thick line) form factor for both components, and using the Casassa for 15DC and the Debye equation for $20 \mathrm{HC}$ (dashed line).

$$
\Gamma(x)=\int_{0}^{\infty} e^{-y} y^{x-1} d y .
$$

Thus by convoluting the ring form factor and Zimm-Schultz distribution [Eqs. (4) and (11)] for a given value of polydispersity $u$, we can estimate the resulting changes on the SANS data. The result of applying such a procedure is shown in Fig. 11 where the form factor $P(Q)$ calculated through Eq. (4) can be seen to be slightly different from that resulting from convolution. One should note that the calculation was carried out for the sample with the highest polydispersity and smaller deviations are expected in all other cases. Furthermore, the difference between fits using Casassa form factors, convoluted form factors and experimental data is within experimental error. Therefore, we conclude that for the samples investigated here, the Casassa form factor adequately fits the experimental SANS data of the cyclic polymers, given the relatively low polydispersity of these samples. In fact the difference between $R_{g, w}^{D}$ values obtained by considering or neglecting the effect of polydispersity is below $10 \%$ for $3 \mathrm{HC}-9 \mathrm{DC}$ and decreases to less than $5 \%$ for the $5 \mathrm{HC}-5 \mathrm{DC}$ sample where the polydispersity of the $\mathrm{H}$ and $\mathrm{D}$ samples are 1.06 and 1.13 , respectively.

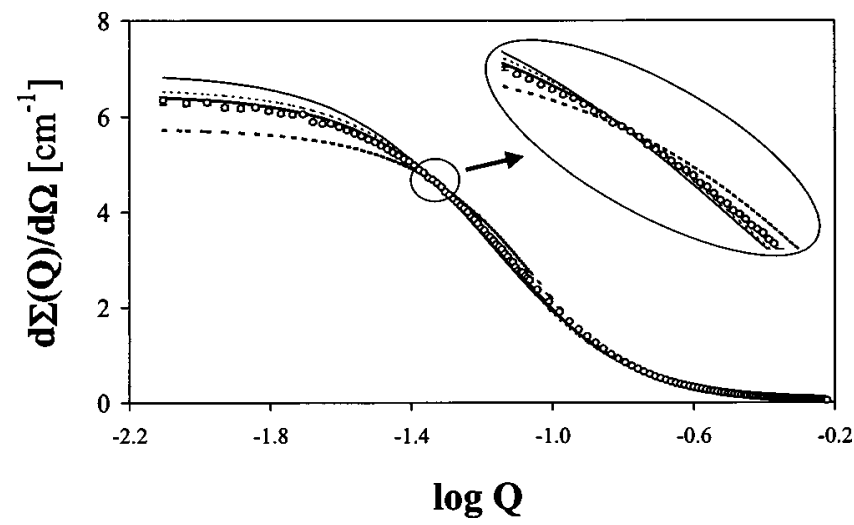

FIG. 14. Experimental data and best fitting obtained for the blend 9HC$11 \mathrm{DC}$ at $\phi_{\mathrm{H}}=0.66$ using either the Debye (thin line) or the Casassa (thick dashed line) form factor for both components, and using the Casassa for $9 \mathrm{HC}$ and the Debye equation for 11DC (thin dashed line) or vice versa (thick line). 
Contrary to cyclic samples with $M_{w} \leqslant 11000$, for high molecular weight cyclic blends, the Casassa form factor does not provide satisfactory fits to the SANS data. This is in part expected, following the considerations earlier with respect to the $\log -\log$ (Fig. 2) and Kratky plots (Figs. 7 and 8) of possible contamination by linear polymer chains of these cyclic fractions. As shown in Figs. 12 and 13, the best fit for the 20HC-15DC blend is obtained using the Debye form factor in Eq. (6) for at least one of the blend components. For the 9HC-11DC blend at dilute conditions $\left(\phi_{\mathrm{H}} \leqslant 0.05\right.$ and $\phi_{\mathrm{H}}$ $\geqslant 0.95$, not shown) fits are good regardless of the model chosen but at $\phi_{\mathrm{H}}=0.26$ (not shown) and 0.66 (Fig. 14) discrepancies are noted when a unique $P(Q)$ function is used for both components. As shown in Fig. 14 the best description of the experimental data is obtained when using the Debye function for the deuterated cyclic PDMS and the Casassa one for its hydrogenous counterpart, or viceversa.

While it could be argued that the results obtained using mixed form factors may not be reliable, they do provide a clear indication of an intermediate scattering behavior between that of a random coil and that of a ring molecule. Results from isotopic blends of cyclic PDMS with $M_{w}$ $\geqslant 11000$ need to be treated carefully as these may be affected by contamination from linear chains or a change or a change in the conformational behavior of rings, with increasing molecular weight. The results shown above indicate that the limit at which cyclic molecules no longer behave as true cyclics is around $M_{w} \approx 11000$.

\section{CONCLUSIONS}

In this paper, we have presented a comparison between the scattering behavior of H/D linear and cyclic mixtures, as a function of molar mass. The use of two different configurations on D22 afforded data over a wide $Q$ range, from 0.0054 to $0.60 \AA^{-1}$ and made it possible to verify that, for the linear PDMS chains, Gaussian scaling is retained up to high $Q$ values. Differences in the $Q$ dependencies of the SANS intensities at high $Q$ s could be detected for the H/D cyclic mixtures, which display a $Q^{(-\nu)}$ dependence, with $\nu$ $>2$. This result supports computer simulations carried out by Muller, Wittmer, and Cates ${ }^{5}$ indicating that the static structure factor $S(Q)$ scales, at high $Q(R g)$ values as the fractal dimension $2.5=1 / 0.4$, in a manner that is consistent with the scaling of the ring dimension as $M^{-\nu}$ with $\nu=0.4$.

As expected on the basis of MC calculations and from the Casassa equation, a pronounced maximum is observed in Kratky plots of cyclics with relatively low molecular weight. Deviations are noted at $M_{w}>11000$ and are attributed to partial contamination of cylic samples with linear chains.

In line with al these observations, the Casassa form factor, provides a good description of the SANS data of the cyclic molecules with $M_{w} \leqslant 11000$, and effect of sample polydispersity are shown to be negligible. Once again deviations are noted for $M_{w}>11000$.

\section{ACKNOWLEDGMENTS}

The authors wish to thank ISIS (UK) and ILL (France) for beam time and the local contacts Dr. Richard Heenan and Dr. Isobelle Grillo for assistance during the experiment. The authors gratefully acknowledge funding from EPSRC to carry out the synthetic and experimental SANS work.

${ }^{1}$ J. S. Higgins, K. Dodgson, and J. A. Semlyen, Polymer 20, 553 (1979). ${ }^{2}$ M. Ragnetti, D. Geiser, H. Hocker, and R. C. Oberthur, Makromol. Chem. 186, 1701 (1985).

${ }^{3}$ J. Roovers, J. Polym. Sci., Polym. Phys. Ed. 23, 1117 (1985).

${ }^{4}$ G. Hadziioannou, P. M. Cotts, G. Tenbrinke, C. C. Han, P. Lutzs, C. Strazielle, P. Rempp, and A. J. Kovacs, Macromolecules 18, 493 (1987).

${ }^{5}$ M. Muller, J. P. Wittmer, and M. Cates, Phys. Rev. E 53, 5063 (1996).

${ }^{6}$ J. Naghizadeh and H. Sotobayashi, J. Chem. Phys. 60, 3104 (1974).

${ }^{7}$ C. J. C. Edwards, D. Rigby, R. F. T. Stepto, K. Dodgson, and J. A. Semlyen, Polymer 24, 391 (1983).

${ }^{8}$ C. J. C. Edwards, D. Rigby, R. F. T. Stepto, and J. A. Semlyen, Polymer 24, 395 (1983).

${ }^{9}$ C. J. C. Edwards, R. W. Richards, R. F. T. Stepto, K. Dodgson, J. A. Higgins, and J. A. Semlyen, Polymer 25, 365 (1984).

${ }^{10}$ C. J. C. Edwards, R. W. Richards, and R. F. T. Stepto, Macromolecules 17, 2147 (1984).

${ }^{11}$ M. E. Cates and J. M. Deutsch, J. Phys. (Paris) 47, 2121 (1986).

${ }^{12}$ T. Pakula and S. Geyler, Macromolecules 21, 1665 (1988).

${ }^{13}$ J. P. Wittmer, W. Paul, and K. Binder, Macromolecules 25, 7211 (1992).

${ }^{14}$ H. L. Frisch, M. Schulz, and P. R. S. Sharma, Comput. Polym. Sci. 4, 13 (1994).

${ }^{15}$ S. Brown and G. Szamel, J. Chem. Phys. 108, 4705 (1998).

${ }^{16}$ S. Brown and G. Szamel, J. Chem. Phys. 109, 6184 (1998).

${ }^{17}$ M. Muller, J. P. Wittmer, and M. E. Cates, Phys. Rev. E 61, 4078 (2000).

${ }^{18}$ S. Brown, T. Lenczycki, and G. Szamel, Phys. Rev. E 63, 052801 (2001).

${ }^{19}$ A. C. Dagger and J. A. Semlyen, Polymer 39, 2621 (1998).

${ }^{20}$ A. C. Dagger and J. A. Semlyen, Polym. Prepr. (Am. Chem. Soc. Div. Polym. Chem.) 39, 579 (1998).

${ }^{21}$ A. C. Dagger and J. A. Semlyen, Polymer 40, 3243 (1999).

${ }^{22}$ V. Arrighi, A. C. Dagger, J. A. Semlyen, and J. S. Higgins, Macromolecules 37, 8057 (2004).

${ }^{23}$ P. J. Flory, J. Chem. Phys. 17, 303 (1949).

${ }^{24}$ J. P. Cotton, D. Decker, H. Benoit et al., Macromolecules 7, 863 (1974).

${ }^{25}$ R. Kirste, W. A. Kruse, and K. Ibel, Polymer 16, 120 (1975).

${ }^{26}$ B. H. Zimm and W. H. Stockmayer, J. Chem. Phys. 17, 1301 (1949).

${ }^{27}$ H. A. Kramers, J. Chem. Phys. 46, 415 (1946).

${ }^{28}$ E. F. Casassa, J. Polym. Sci. A 3, 605 (1965).

${ }^{29}$ W. Burchard and M. Schmidt, Polymer 21, 745 (1980).

${ }^{30}$ J. Chojnwski and L. Wilczek, Makromol. Chem. 180, 117 (1979).

${ }^{31}$ J. A. Semlyen, Cyclic Polymers (Elsevier Applied Science, London, 1986), Chap. 3.

${ }^{32}$ K. Dodgson, D. Sympson, and J. A. Semlyen, Polymer 19, 1285 (1978).

${ }^{33}$ S. M. King, Using Colette, A Simple Guide (ISIS Facility, Didcot, Oxford, 1997).

${ }^{34}$ P. G. deGennes, Scaling Concepts in Polymer Physics (Cornell University Press, Ithaca, NY, 1979)

${ }^{35}$ K. Binder, J. Chem. Phys. 79, 6387 (1983).

${ }^{36}$ G. S. Greschner, Makromol. Chem. 170, 203 (1973).

${ }^{37}$ O. Kratky, Pure Appl. Chem. 12, 483 (1966).

${ }^{38}$ J. S. Higgins and H. C. Benoit, Polymers and Neutron Scattering (Oxford University Press, Oxford, 1993).

${ }^{39}$ D. Y. Yoon and P. J. Flory, Polymer 16, 645 (1975).

${ }^{40}$ M. Rawiso, R. Duplessix, and C. Picot, Macromolecules 20, 630 (1987).

${ }^{41}$ G. Beaucage, S. Sukumaran, S. J. Clarson, M. S. Kent, and D. W. Schaefer, Macromolecules 29, 8349 (1996).

${ }^{42}$ J. Kugler, E. W. Fischer, M. Peuscher, and C. D. Eisenbach, Makromol. Chem. 184, 2325 (1983).

${ }^{43}$ G. V. Schultz, Z. Phys. Chem. Abt. B 43, 25 (1939).

${ }^{44}$ B. H. Zimm, J. Chem. Phys. 16, 1099 (1948). 